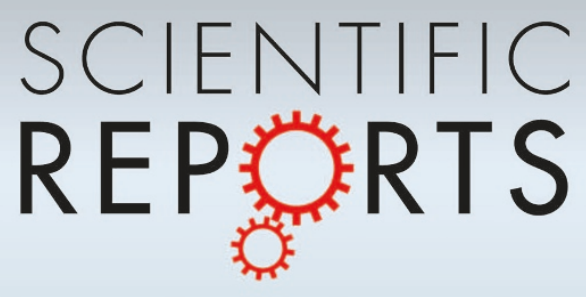

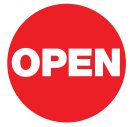

SUBJECT AREAS:

CHEMICAL PHYSICS

THEORETICAL CHEMISTRY

PHYSICAL CHEMISTRY

ATOMIC AND MOLECULAR

PHYSICS

Received

7 August 2012

Accepted

14 November 2012

Published

5 December 2012

Correspondence and requests for materials should be addressed to Y.-P.Z. (yzhao@imech. ac.cn)

\section{Capillary wave propagation during the delamination of graphene by the precursor films in electro-elasto-capillarity}

\author{
Xueyan Zhu, Quanzi Yuan \& Ya-Pu Zhao
}

State Key Laboratory of Nonlinear Mechanics, Institute of Mechanics, Chinese Academy of Sciences, Beijing 100190, China.

Molecular dynamics simulations were carried out to explore the capillary wave propagation induced by the competition between one upper precursor film $(\mathrm{PF})$ on the graphene and one lower PF on the substrate in electro-elasto-capillarity (EEC). During the wave propagation, the graphene was gradually delaminated from the substrate by the lower PF. The physics of the capillary wave was explored by the molecular kinetic theory. Besides, the dispersion relation of the wave was obtained theoretically. The theory showed that the wave was controlled by the driving work difference of the two PFs. Simulating the EEC process under different electric field intensities $(E)$, the wave velocity was found insensitive to $E$. We hope this research could expand our knowledge on the wetting, electrowetting and EEC. As a potential application, the electrowetting of the PF between the graphene and the substrate is a promising candidate for delaminating graphene from substrate.

lectrowetting $(\mathrm{EW})^{1}$, in which electric field is applied to modify the wetting properties ${ }^{2-6}$ of the substrate, is important for a variety of applications including micro/nano droplet manipulation ${ }^{7}, E$ display $^{8}$, lab on chip ${ }^{9}$, etc. While much progress has been made, most of the studies on EW were on rigid and fixed substrate. However, the wetting phenomena on soft and movable substrate, known as elasto-capillarity (EC) ${ }^{10}$, are more common both in engineering and biological systems, such as the stiction of micromachined cantilever beams in wet conditions ${ }^{11}$ or the closing hermetically of Nymphoides flowers when sink into water ${ }^{12}$. EC has been used to produce capillary origami of different shapes in experiments ${ }^{13}$ and Král group's pioneer work predicted that a nanodroplet could guide the graphene to fold by molecular dynamics (MD) simulations ${ }^{14}$. To realize the controllable and reversible process of EC, Zhao group has applied electric field in EC, and named the combination of EW and EC the electro-elasto-capillarity $(\mathrm{EEC})^{15,16}$. EEC deserves to be concerned of both science and applications. When placed on a soft substrate, a droplet, whose size is larger than the EC length ${ }^{17}$, can be spontaneously wrapped under the vertical component of surface tension and released by applying the electric field. The capillary force, elastic force and electric force compete in EEC. At the atomic scale, the precursor film (PF) produced by the droplet would interact with the soft substrate to unwrap the folding structure. EEC has been used to realize the revisable folding of capillary origami both in experiments ${ }^{16,18}$ and 2D MD simulations ${ }^{15}$. The controllable folding structure promises to be a good candidate in encapsulation and targeted releasing of drug, eliminating evaporation of small droplet ${ }^{16}$, improving the performance of photovoltaic systems ${ }^{19}$, etc. In this paper, we not only realized the EEC process by MD simulations, but also found the capillary wave propagation induced by the competition between two PFs during this process.

At nanoscale, the folding structure is pushed by the PF to unwrap during EEC as shown in Fig. 1b. PF, first proposed by $\mathrm{Hardy}^{20}$, is usually a molecular layer propagating ahead of the nominal contact line. When viewed at the atomic scale, the PF plays an important role in the dynamic wetting process on hydrophilic substrate. Besides, during EW, due to the long-ranged Coulomb interaction induced by the electric field, the ionized or polar deionized liquid molecules can also be attracted to the substrate to form the PF. The existence of the PF has been verified by numerous experiments ${ }^{21-26}$ and MD simulations ${ }^{15,27}$. Through theoretical analysis, de Gennes predicted that the PF was controlled by the disjoining pressure ${ }^{28}$. Previous studies were about single PF on rigid substrate. But there was no report on the coupled interaction between multilayer PFs to the best of our knowledge. In our work, during EEC, two PFs were formed as shown in Fig. 2c (the upper PF on the soft substrate and the lower PF on the rigid substrate). Through MD and theoretical analysis, we found that the two PFs competed to form the capillary wave at nanoscale. And this capillary wave was explored by molecular kinetic theory (MKT) in the atomic level for the first time. 

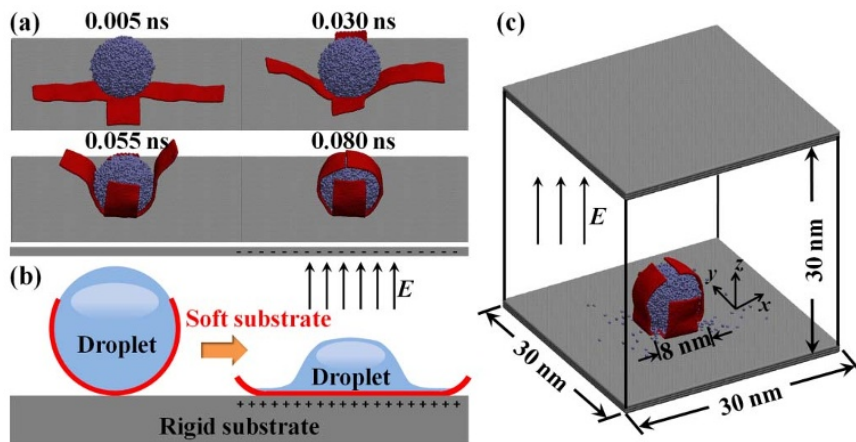

Figure $1 \mid$ Molecular dynamics (MD) simulation domain of elastocapillarity (EC) and electro-elasto-capillarity (EEC). (a) The dynamic EC process of a water droplet wrapped by the graphene. (b) Schematic diagram of the unwrapping process of the folding structure pushed by the precursor film (PF) under the electric field. (c) MD simulation domain of EEC. Silver, red and ice blue atoms represent graphite, graphene and water atoms, respectively.

In this paper, graphene was chosen as the soft substrate. A variety of applications has been spurred by graphene for its unique properties $^{29-32}$. The key challenge for realizing these applications is the ability to produce and transfer graphene. Large area growth of graphene is on a substrate such as chemical vapor deposition on $\mathrm{Pt}^{33}$ or $\mathrm{Cu}^{34}$. However, due to the extremely large adhesion energy of graphene on the substrate ${ }^{35}$, delaminating and transferring graphene from the substrate is usually difficult. Graphene has been transferred by directly etching the substrate ${ }^{36}$. But, exposed to the etching solution, graphene is inevitably contaminated. In this paper, we found a candidate method for separating the graphene from the substrate without damage by the PF during the wave propagation.

We carried out MD simulations to realize the dynamic EEC process. Under the electric field, some water molecules were attracted to the graphene and others to the substrate to form the two PFs. The upper PF propagated to unwrap the folding graphene and the lower $\mathrm{PF}$ propagated to delaminate the graphene from the substrate, which was driven by the Maxwell stress as shown in Fig. $4 \mathrm{~b}$. During this

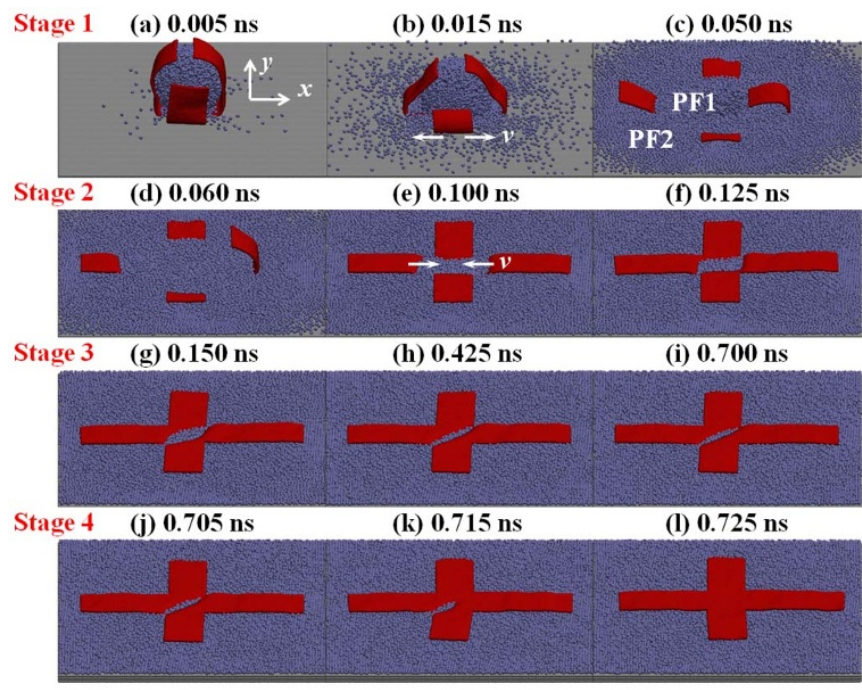

Figure $2 \mid$ Capillary wave propagation (borderline between red and ice blue) induced by the competition between PF1 and PF2 during which the graphene was delaminated from the substrate by PF2. (a-c) stage 1: wave propagating along the negative direction. $(\mathrm{d}-\mathrm{f})$ stage 2 : wave propagating along the positive direction. (g-i) stage 3: formation of a stable wrinkle. $(j-1)$ stage 4 : disappearance of the wrinkle.

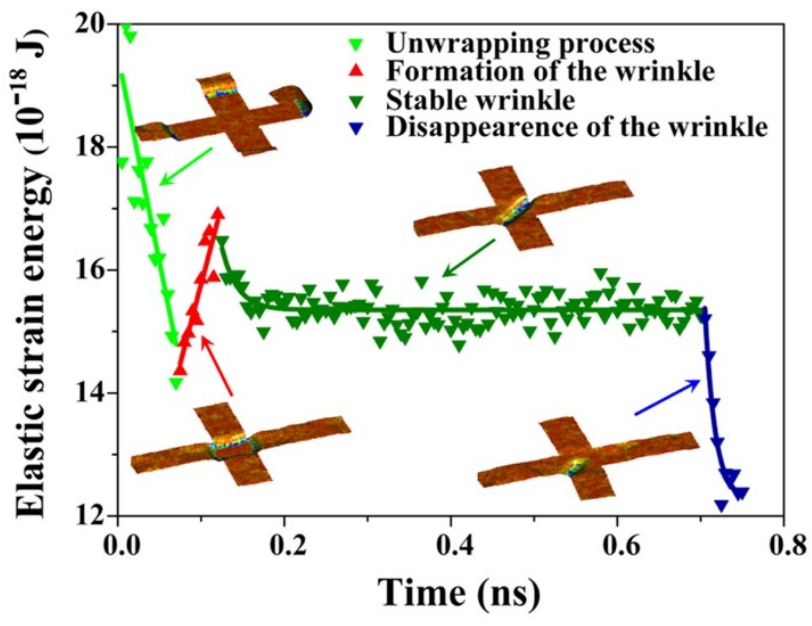

Figure 3 The variation of the elastic strain energy of the graphene with respect to time. The inset describes the distribution of the elastic strain energy of the graphene in which the energy gradually decreases from blue to red.

process, we found the capillary wave propagation induced by the competition between the two PFs. The propagation of the capillary wave was explored by MKT. The theory and the simulations qualitatively agreed well on the velocity. From the perspective of wave dynamics, a scaling analysis was made to obtain the dispersion relation and the average wave velocity, which was of the same order with that in the simulations. From the theoretical analysis above, we concluded that the wave propagation was controlled by the driving work difference of the two PFs, while the energy was mainly dissipated by the viscosity. In addition, a series of EEC simulations under different electric field intensities $(E)$ and different boundary conditions showed that the capillary wave velocity was insensitive to $E$ and the boundary. It is expected that our work can provide valuable guidance for utilizing the PF to unwrap the folding structure or delaminate graphene from substrate.

\section{Results}

First, a water droplet was placed on the graphene without electric field. Under the vertical component of surface tension, the droplet was spontaneously wrapped by the graphene in 0.08 ns (Fig. 1a)

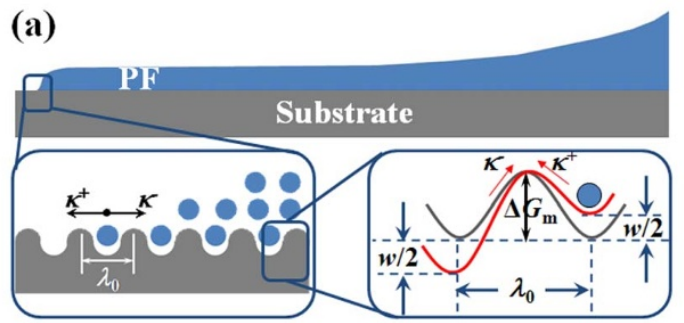

(b)
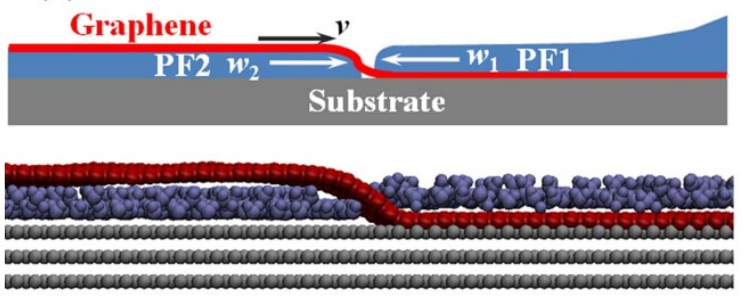

Figure $4 \mid$ The atomic theory of the interaction between two PFs. (a) Schematic diagram of the molecular kinetic theory (MKT). (b) The competition between two PFs. 
corresponding to the viscous time $t_{\mathrm{vis}} \sim l /\left(\gamma / \eta_{\mathrm{w}}\right) \sim 0.1 \mathrm{~ns}$, where $\eta_{\mathrm{w}}$ $=0.001 \mathrm{~Pa} \cdot \mathrm{s}$ is the viscosity of bulk water, $l=8 \mathrm{~nm}$ the droplet diameter and $\gamma=0.072 \mathrm{~N} / \mathrm{m}$ the surface tension. To release the droplet, an electric field was applied (Fig. 1b). During the releasing process, the elastic energy decreased, while the interface energy increased. Here, the folding graphene was pushed by the PF to unwrap when $E \sim 1 \mathrm{~V} / \mathrm{nm}$. In the following results and discussions, $E=3 \mathrm{~V} / \mathrm{nm}$ (Fig. 1c).

During EEC, PF spread on the soft graphene and the fixed graphite at the same time (Fig. 2). The upper PF on the graphene and the lower $\mathrm{PF}$ on the graphite were distinguished by $\mathrm{PF} 1$ and PF2, respectively (Fig. 2c). The competition between PF1 and PF2 induced the capillary wave propagation. The expanding wave velocity was defined to be negative (Fig. $2 \mathrm{~b}$ ) and the contracting wave velocity (Fig. 2e) to be positive. The whole EEC process was divided into four stages. In the first stage (Fig. $2 \mathrm{a}-\mathrm{c}$ ), the wave propagated along the negative direction and quickly accelerated in $0.015 \mathrm{~ns}$. During this period, the folding graphene was unwrapped and pushed by PF1 with a force of the order of $10 \mathrm{nN} / \mathrm{nm}$. Contemporarily, some water molecules were attracted to the graphite and formed PF2. When the disjoining pressure of PF2 was larger than that of PF1, the wave started slowing down and countermoved at 0.05 ns. In the second stage (Fig. 2d-f), PF2 drilled into the confined space between the graphene and the graphite driven by the Maxwell stress (see Supplementary Information 7), while PF1 started receding from the graphene. During this process, the capillary wave propagated along the positive direction and accelerated from 0.06 to $0.1 \mathrm{~ns}$. Then the waves in the four flakes of the graphene encountered in the center and slowed down. In the third stage (Fig. 2g-i), a stable wrinkle formed in the graphene under the external pressure from PF2. The wrinkle remained for about $0.5 \mathrm{~ns}$. It should be noted that the wrinkle was slowly shrinking, which was hard to be detected, while the curvature of the wrinkle simultaneously increased. In the fourth stage (Fig. $2 j-$ 1), the wrinkle quickly disappeared pressed by PF2. In the end, PF2 completely separated the graphene from the substrate (Fig. 2l). The wave propagation during EEC was also accompanied by the variation of the elastic strain energy of the graphene with respect to time.

The elastic strain energy of the graphene oscillated to decrease during EEC (Fig. 3). In the first stage, owing to the unwrapping of the folding graphene, the elastic strain energy decreased (shallow green line in Fig. 3). Then, pushed by PF2, the graphene was wrinkled, following which the four waves encountered in the center. This led to the increase of the elastic strain energy in the second stage (red line in Fig. 3). After a short relaxation, the elastic strain energy stabilized at $1.55 \times 10^{-17} \mathrm{~J}$, corresponding to the stable wrinkle of the third stage (deep green line in Fig. 3), which lasted for about $0.5 \mathrm{~ns}$. In the fourth stage, the wrinkle disappeared, which resulted in the decrease of the elastic strain energy (blue line in Fig. 3). It should be noted that the elastic strain energy was mainly stored in the wrinkle, which was induced by the competition between PF1 and PF2, as shown in the inset of Fig. 3.

\section{Discussion}

In the atomic level, we developed MKT proposed by Eyring et $a^{37}$ to analyze the capillary wave propagation during EEC. In this theory, the motion of the contact line is determined by the adsorption and desorption of the fluid molecules on the surface sites separated by a distance $\lambda_{0}\left(\lambda_{0}=2.46 \AA\right.$ for graphene or graphite $)$ as shown in Fig. 4 a. For the surface sites with energy barrier of $\Delta G_{\mathrm{m}}$, the equilibrium jump frequency of the fluid molecules $\kappa^{0}=\left(k_{\mathrm{B}} T / h\right) \exp \left(-\Delta G_{\mathrm{m}} /\right.$ $\left.k_{\mathrm{B}} T\right)$, where $k_{\mathrm{B}}$ and $h$ are the Boltzmann and Planck constant, respectively. The contact line would move if the adsorption equilibria are disturbed by the external driving work. In the case of EW, the driving work for the spreading droplet contains the van der Waals interactions per unit area $w_{\mathrm{v}}$, the polar interactions between water molecules $w_{\mathrm{p}}$, the different structure of the PF from the bulk liquid $w_{\mathrm{s}}{ }^{38}$ and additional average electric energy $w_{\mathrm{E}}$. So the total work that induces the motion of the contact line is $w=w_{\mathrm{v}}+w_{\mathrm{p}}+w_{\mathrm{s}}+w_{\mathrm{E}}{ }^{15}$. For water droplet of nanoscale, the electric energy describing the interaction between the electric field and the droplet is $w_{\mathrm{E}} \sim \sum_{i}\{-|\overrightarrow{\mathrm{E}}| \times$ $\left.\left|\vec{\mu}_{\mathrm{i}}\right| \times L\left[|\overrightarrow{\mathrm{E}}| \times\left|\vec{\mu}_{\mathrm{i}}\right| /\left(k_{\mathrm{B}} T\right)\right]\right\}$, where $\vec{E}$ is the electric field vector, $\vec{\mu}_{\mathrm{i}}$ the electric dipole moment and $L(x)=\operatorname{coth}(3 x)-1 /(3 x)$ the Langevin function. All the forces that induced the driving work of the PF were contained into the disjoining pressure ${ }^{39}$, which could be obtained directly from MD results. In our simulations, the capillary wave propagation during EEC was induced by the competition between PF1 and PF2 (Fig. 4b). So the wave velocity can be calculated by

$$
v=2 \kappa^{0} \lambda_{0} \sinh \frac{w_{2}-w_{1}}{2 n k_{\mathrm{B}} T},
$$

where $w_{1}$ is the driving work per unit area of PF1, $w_{2}$ that of PF2 and $n$ the number of adsorption sites per unit area $\left(n=0.19 \AA^{-2}\right.$ for graphene or graphite). This is the governing equation of the competition between two PFs, in which $w_{2}-w_{1}$ and $\kappa^{0}$ are variables.

Here, we discussed the wave in the left flake of the graphene (Fig. 4b). The wave propagating along the right direction was defined to be positive. As shown in Fig. 5a, $w_{1}$ was monotonically decreasing in the first $0.05 \mathrm{~ns}$; then stabilized at about $2.5 \mathrm{~J} / \mathrm{m}^{2}$. Oppositely, $w_{2}$ was monotonically increasing in the first $0.05 \mathrm{~ns}$; then stabilized at about $3.5 \mathrm{~J} / \mathrm{m}^{2}$. Before $0.05 \mathrm{~ns}, w_{2}<w_{1}$, which resulted in the wave propagating along the negative direction in the first stage (Fig. 2a-c). At $0.05 \mathrm{~ns}, w_{2}=w_{1}$, substituting which into equation (1), we obtained zero velocity. The wave countermoved at this moment. In the second stage, $w_{2}>w_{1}$, the wave propagated along the positive direction (Fig. $2 \mathrm{~d}-\mathrm{f}$ ). Then $w_{2}-w_{1}$ stabilized at about $1 \mathrm{~J} / \mathrm{m}^{2}$ in the third stage (Fig. $2 \mathrm{~g}-\mathrm{i}$ ). Considering the wrinkle area be about 1000 $\AA^{2}$, the stable work difference originating from $w_{2}-w_{1}$ was evaluated to be of the order of $10^{-17} \mathrm{~J}$, which had the same order with the elastic strain energy stored in the stable wrinkle (deep green line in Fig. 3). From the analysis, we inferred that $w_{2}-w_{1}$ transferred to the elastic strain energy of the graphene wrinkle in the end.

To obtain the wave velocity from equation (1), the driving work difference $w_{2}-w_{1}$ and the equilibrium jump frequency $\kappa^{0}$ should be obtained in advance. The driving work difference between PF2 and $\mathrm{PF} 1$, which drove the wave, was obtained from $w_{2}$ and $w_{1}$ illustrated above (Fig. 5a). As shown in Fig. 5b, $w_{2}-w_{1}$ was negative before $0.05 \mathrm{~ns}$, which drove the wave propagating along the negative direction; then positive from $0.05 \mathrm{~ns}$ to $0.14 \mathrm{~ns}$, which drove the wave propagating along the positive direction. After $0.14 \mathrm{~ns}, w_{2}-w_{1}$ stabilized at about $1 \mathrm{~J} / \mathrm{m}^{2}$, which transferred to the elastic strain energy stored in the stable wrinkle. We fitted $w_{2}-w_{1}$ by exponential function of the red line as shown in Fig. 5b.

Then, we endeavored to obtain the equilibrium jump frequency $\kappa^{0}$. From Blake's theory ${ }^{40}$, the fluid molecules within the contact line are hindered not only by interactions with solid surface (adhesion), but also by viscous interactions with neighboring fluid molecules. So the energy barrier $\Delta G_{\mathrm{m}}=\Delta G_{\mathrm{a}}+\Delta G_{\mathrm{v}}$, where $\Delta G_{\mathrm{a}}$ and $\Delta G_{\mathrm{v}}$ are the contributions from adhesion and viscosity, respectively. According to Eyring's theory ${ }^{37}$, the relationship between the viscosity and $\Delta G_{\mathrm{v}}$ is $\eta$ $=\left(h / v_{\mathrm{L}}\right) \exp \left(\Delta G_{\mathrm{v}} / k_{\mathrm{B}} T\right)$, where $\eta$ is the viscosity and $v_{\mathrm{L}}$ the volume of the unit of flow. Thus, the equilibrium jump frequency can be obtained

$$
\begin{aligned}
\kappa^{0} & =\frac{k_{\mathrm{B}} T}{h} \exp \left(-\frac{\Delta G_{\mathrm{m}}}{k_{\mathrm{B}} T}\right)=\frac{k_{\mathrm{B}} T}{h} \exp \left(-\frac{\Delta G_{\mathrm{a}}+\Delta G_{\mathrm{v}}}{k_{\mathrm{B}} T}\right) \\
& =\frac{k_{\mathrm{B}} T}{\eta v_{\mathrm{L}}} \exp \left(-\frac{\Delta G_{\mathrm{a}}}{k_{\mathrm{B}} T}\right) .
\end{aligned}
$$

In our work, the viscosity of the PF is $\eta=0.90 \times 10^{-2} \mathrm{~Pa} \cdot \mathrm{s}$ (see Supplementary Information 1) and the volume of the water molecule is $v_{\mathrm{L}}=34.22 \mathrm{~A}^{3}$. We assumed that the adhesion energy contributing 

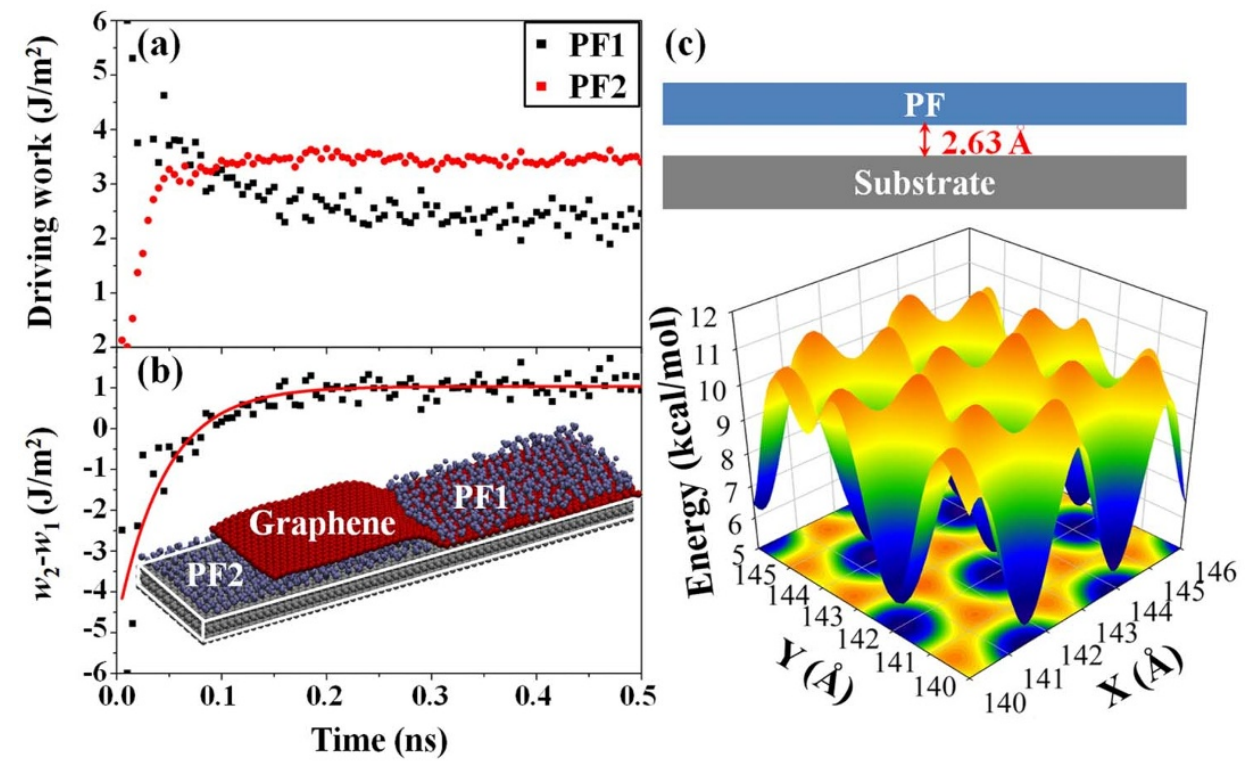

Figure $5 \mid$ (a) Driving work per unit area of the two PFs from the MD simulations. (b) Driving work difference of the two competing PFs. The inset represents the competition between two PFs from the simulations. (c) Potential surface of the interaction between the PF and the substrate. The red colour represents high energy, whereas the blue colour represents low energy.

to the competition between the two PFs $\Delta G_{\mathrm{a}}=\left(\Delta G_{\mathrm{a} 1}+\Delta G_{\mathrm{a} 2}\right) / 2$, where $\Delta G_{\mathrm{a} 1}$ is the work of adhesion between a water molecule and the graphite, and $\Delta G_{\mathrm{a} 2}$ is that between a water molecule and the graphene. Considering the Lennard-Jones (LJ) potential used in our simulations,

$$
\begin{aligned}
\Delta G_{\mathrm{a} 1} & =\frac{4 \pi \varepsilon \sigma^{3} \rho_{\mathrm{v}}}{3}\left(\frac{\sigma^{3}}{2 h_{1}^{3}}-\frac{\sigma^{9}}{15 h_{1}^{9}}\right) \\
\Delta G_{\mathrm{a} 2} & =4 \pi \varepsilon \sigma^{2} \rho_{\mathrm{s}}\left(\frac{\sigma^{4}}{2 h_{2}{ }^{4}}-\frac{\sigma^{10}}{5 h_{2}^{10}}\right),
\end{aligned}
$$

where $\varepsilon=0.15 \mathrm{kcal} / \mathrm{mol}$ and $\sigma=3.39 \AA$ are the LJ parameters, $\rho_{\mathrm{v}}=$ $0.11 \AA^{-3}$ and $\rho_{\mathrm{s}}=0.38 \AA^{-2}$ are the number of carbon atoms per unit volume of graphite and per unit area of graphene, respectively, $h_{1}=$ $2.91 \AA$ and $h_{2}=3.39 \AA$ are the equilibrium distance between a water molecule and graphite and that between a water molecule and graphene, respectively. By substituting all the parameters into equation (3), $\Delta G_{\mathrm{a}}=\left(\Delta G_{\mathrm{a} 1}+\Delta G_{\mathrm{a} 2}\right) / 2=2.00 \mathrm{kcal} / \mathrm{mol}$. Then, after obtaining $\kappa^{0}=0.47 \mathrm{~ns}^{-1}$ from equation (2), the energy barrier could be derived by $\Delta G_{\mathrm{m}}=k_{\mathrm{B}} T \ln \left(k_{\mathrm{B}} T / \kappa^{0} h\right)=5.66 \mathrm{kcal} / \mathrm{mol}$. So the energy dissipation from the viscosity $\Delta G_{\mathrm{v}}=\Delta G_{\mathrm{m}}-\Delta G_{\mathrm{a}}=3.66 \mathrm{kcal} / \mathrm{mol}$. From the data obtained, $\Delta G_{\mathrm{v}}>\Delta G_{\mathrm{a}}$, which illustrated that the energy was mainly dissipated by the viscosity. If we regarded the energy barrier as the height of the potential well above the substrate, the effective equilibrium distance between the PF and the substrate $h_{\mathrm{e}}=2.63 \AA$ (see Supplementary Information 2). The potential surface was scanned at $h_{\mathrm{e}}$ above the substrate as shown in Fig. 5c.

Using $w_{2}-w_{1}$ and $\kappa^{0}$, we obtained the velocity from equation (1). But this velocity was not the wave velocity, because $w_{2}-w_{1}$ also contained the energy that drove the expanding spreading of the PFs and the energy stored in the graphene wrinkle. So we removed this part by subtracting the velocity function, which fitted from the part of the velocity after $0.14 \mathrm{~ns}$ when the wave frozen. Then the wave velocity function was obtained. The theory proposed here agreed qualitatively with the simulations as shown in Fig. 6. The deviation was due to the fact that MKT was a quasi-static theoretical model. Other factors, i.e., the PF which was not spreading in the wave direction, the effect of the graphene on the PF, and etc, were not considered in this theory. The velocity graph plotted here also corresponded to the four stages (Fig. 2) illustrated in the results part.
Using the scaling theory ${ }^{41}$ from the view of energy equilibrium, we derived the dispersion relation of the capillary wave to evaluate the phase velocity. Newtonian fluid model was used and the flow was assumed to be incompressible. For the whole system, the energy generated by the driving work difference $\Delta w=w_{2}-w_{1}$ equaled the energy dissipated viscously by the PF. Equating the energy input and dissipation, we obtained the dispersion relation of the wave (see Supplementary Information 3)

$$
\omega \sim \frac{\Delta w k^{2} d_{0}^{2}}{\eta h}
$$

where $k=2 \pi / \lambda$ is the wave number, $d_{0}$ the thickness of the PF, $\eta$ the viscosity of the PF and $h$ the amplitude of the wave. Then the phase velocity of the wave ${ }^{42}$ was derived

$$
v=\frac{\omega}{k} \sim \frac{\Delta w k d_{0}}{\eta h / d_{0}} .
$$

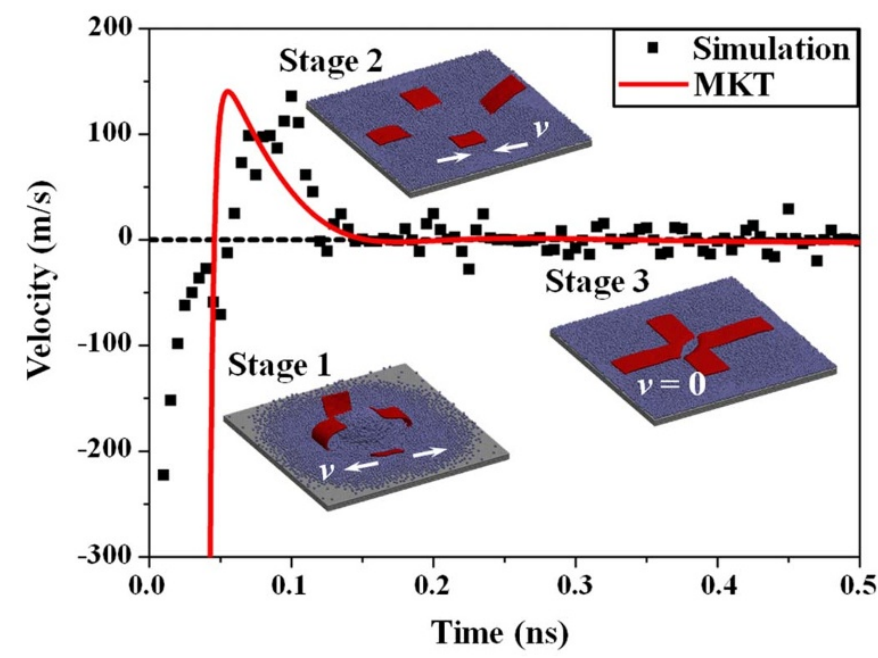

Figure 6 | The capillary wave velocity obtained from MD simulations and MKT, respectively. The inset shows the corresponding stage of the wave propagation. 
Table 1 | The average capillary wave velocity $v$ with respect to the electric field intensity $E$

\begin{tabular}{|c|c|c|c|c|c|c|c|c|c|}
\hline $\begin{array}{l}E(\mathrm{~V} / \mathrm{nm}) \\
V(\mathrm{~m} / \mathrm{s})\end{array}$ & $\begin{array}{r}2.00 \\
95.91\end{array}$ & $\begin{array}{r}2.50 \\
83.03\end{array}$ & $\begin{array}{r}2.70 \\
95.99\end{array}$ & $\begin{array}{r}3.00 \\
86.07\end{array}$ & $\begin{array}{r}3.30 \\
86.01\end{array}$ & $\begin{array}{r}3.60 \\
100.55\end{array}$ & $\begin{array}{r}3.90 \\
70.80\end{array}$ & $\begin{array}{r}4.30 \\
86.16\end{array}$ & $\begin{array}{r}4.60 \\
80.77\end{array}$ \\
\hline
\end{tabular}

If the dispersion effect was not considered, the relation $\lambda=2 \pi / k=$ $2 \pi d_{0}^{2} / h$ must be satisfied. So equation (5) could be further simplified to

$$
v \sim \frac{\Delta w}{\eta}
$$

This equation illustrated that the propagation of the wave was driven by the competition between $\Delta w$ and the viscosity. Equation (6) had the same form with the capillary velocity $\gamma / \eta^{43}$, which described the competition between surface tension and viscosity without electric field.

The phase velocity calculated from equation (6) was the average wave velocity. So the driving work difference $\Delta w$ (Fig. 5b) should also be averaged over time to evaluate the wave velocity from equation (6). Without loss of generalization, $\Delta w$ was averaged from $0 \mathrm{~ns}$ to $0.05 \mathrm{~ns}$ of the first stage, during which the wave propagated along the negative direction. $\overline{\Delta w}=\left|\int\left(w_{2}-w_{1}\right) d t / \Delta t\right|=2.50 \mathrm{~J} / \mathrm{m}^{2}$. On the other hand, the viscosity of the PF was $\eta \sim 10^{-2} \mathrm{~Pa} \cdot \mathrm{s}$ (see Supplementary Information 1). From equation (6), the average wave velocity was obtained to be of the order of $100 \mathrm{~m} / \mathrm{s}$, which had the same order with that $(\sim 86.07 \mathrm{~m} / \mathrm{s})$ from the MD results. We also noticed that the capillary velocity of the bulk water without electric field was $\gamma / \eta_{\mathrm{w}} \sim 100 \mathrm{~m} / \mathrm{s}$. Besides, from the dispersion relation of the capillary wave without electric field, the wave velocity $v=(\gamma k / \rho)^{1 / 2} \sim$ $100 \mathrm{~m} / \mathrm{s}$, where $k \sim 10^{9} \mathrm{~m}^{-1}, \rho=1000 \mathrm{~kg} / \mathrm{m}^{3}$.

The analysis above was based on the EEC process under $E=3 \mathrm{~V} /$ $\mathrm{nm}$. However, we found that if only $E$ was larger than a critical value (approximately $1 \mathrm{~V} / \mathrm{nm}$ ), PF2 could drill into the confined space between the graphene and the substrate, and compete with PF1 to form the capillary wave (Fig. $4 \mathrm{~b}$ ). To explore the relation between the capillary wave velocity and $E$, we had done a series of EEC simulations under different $E$. Table 1 showed the change of the average wave velocity with respect to $E$. Interestingly, the capillary wave velocity was insensitive to $E$. In addition, we also found the propagation of the capillary wave in another EEC model, in which one end of the graphene was fixed (see Supplementary Information 5).

Previous discussions were based on MD simulations with periodic boundary condition (PBC). Although the PBC has the advantage that it is efficient, it has the defect that the periodic system would interact at the boundary. So is the wave cased by the PBC and strongly dependent on the boundary? To address this question, we have established another model in Supplementary Information 9. In this model, the boundary condition was set to be non-periodic and the substrate large enough to ensure that the boundary had little impact on the graphene-water system. In addition, the simulation box was set to be larger than the substrate. We not only found the capillary wave in this model, but also calculated the wave velocity to be of the order of $100 \mathrm{~m} / \mathrm{s}$. Therefore, the wave reported in this paper was not induced by the PBC, but by the competition between two PFs as was illustrated above. And the wave velocity was insensitive to the boundary.

In summary, the capillary wave propagation at nanoscale, induced by the competition between two PFs during EEC, was found and studied for the first time. During this process, the PF could separate the graphene from the substrate by EW between the graphene and the substrate driven by the Maxwell stress. This phenomenon can be a prospect candidate for delaminating graphene. From the atomic level, we developed MKT to describe the competition between two PFs. Then a scaling analysis was carried out to obtain the dispersion relation and average wave velocity. The theory showed that the competition between two PFs was controlled by their driving work difference originating from the disjoining pressure and the viscosity took priority in the energy dissipation. Simulating the EEC process under different $E$ and different boundary conditions, the capillary wave velocity was found insensitive to $E$ and the boundary. In addition, multilayer PFs were found during EEC (see Supplementary Information 4). The behavior of the PFs obeyed the power law with respect to time. The friction on the PF was larger than that on the substrate. We hope this work could provide valuable guidance for utilizing the PF to unwrap the folding structure or delaminate graphene from substrate.

\section{Methods}

MD simulations implemented in LAMMPS ${ }^{44}$ were carried out in this article. Water molecules and carbon atoms were modeled as LJ particles with truncated Coulomb potential interaction if charged

$$
V_{i j}=4 \varepsilon_{i j}\left[\left(\frac{\sigma_{i j}}{r}\right)^{12}-\left(\frac{\sigma_{i j}}{r}\right)^{6}\right]+\frac{C q_{i} q_{j}}{r},
$$

where $\varepsilon_{i j}$ is the potential well depth when there are no charges, $\sigma_{i j}$ the distance at which the LJ potential is zero, $C$ the Coulomb constant, $q_{i}$ and $q_{j}$ the charges on the two atoms, $r$ the distance between $i$ and $j$ atoms. The LJ and Coulomb cutoff were set to be $10 \AA$ and $9.5 \AA$, respectively. The LJ parameters came from the consistent valence force-field (cvff) ${ }^{45}$, which is based on the experimental values and ab initio calculations. The interactions between water molecules and carbon atoms were calculated by the Lorentz-Berthelot (LB) rule:

$$
\begin{aligned}
\varepsilon_{i j} & =\sqrt{\varepsilon_{i i} \varepsilon_{j j}} \\
\sigma_{i j} & =\left(\sigma_{i i}+\sigma_{j j}\right) / 2 .
\end{aligned}
$$

Moreover, we set the interaction between the graphene and the substrate by $1 / 100$ smaller than the calculated values by equation (8), to avoid the graphene sticking to the substrate during EC. To evaluate the precision of the LJ potential parameterized by the LB rule for simulating graphene-water interfacial dynamics, we have performed density functional theory simulations in Supplementary Information 8 for making a comparison. The results showed that the LJ potential parameterized by the LB rule could simulate the interactions between graphene and waters within acceptable precision.

The extended single point charge (SPC/E) water model ${ }^{46}$ was used. The SPC/E model was established by adding an average polarization correction to the single point charge (SPC) model, with a modified value of $q_{\mathrm{O}}$ (charge on oxygen atom) and $q_{\mathrm{H}}$ (charge on hydrogen atom). Compared with SPC, transferable intermolecular potential 3 point (TIP3P), transferable intermolecular potential 4 point (TIP4P) and transferable intermolecular potential 5 point (TIP5P) water model, SPC/E model predicts a better density, diffusion coefficient, surface tension and viscosity, which govern the EEC process, compared to the experimental values.

In order to model the electric field, the first layer of the graphite and the above graphite layer were charged with positive and negative charges, respectively, as shown in Fig. 1c. The field intensity could be calculated by $E=Q /\left(A \varepsilon_{0} \varepsilon\right)$ along the $z$ direction, where $Q, A, \varepsilon_{0}$ and $\varepsilon$ represent the charges on each layer, the area of the layer, the vacuum permittivity and the relative permittivity of water, respectively. The calculated electric field intensity was $E \sim 1 \mathrm{~V} / \mathrm{nm}$.

The whole system was modeled in NVT ensemble with Nose/Hoover method at $300 \mathrm{~K}$ and the time step was $1 \mathrm{fs}$. The boundary was periodic in three dimensions. And the box size was $30 \mathrm{~nm} \times 30 \mathrm{~nm} \times 30 \mathrm{~nm}$. The droplet diameter was $8 \mathrm{~nm}$ containing 8904 water molecules (Fig. 1c). At the initial state, the graphene was placed on the center of the substrate, then the water droplet on the graphene. The substrate was fixed during the whole process. After relaxing for $0.1 \mathrm{~ns}$, the droplet was wrapped by the graphene. Then we applied the electric field. The whole EEC process was simulated for $0.8 \mathrm{~ns}$. The same process under different field intensities was repeated.

1. Mugele, F. \& Baret, J. C. Electrowetting: from basics to applications. J. Phys.: Condens. Matter 17, R705-R774 (2005).

2. Wang, B. Y. \& Král, P. Chemically tunable nanoscale propellers of liquids. Phys. Rev. Lett. 98, 266102 (2007).

3. Bonn, D., Eggers, J., Indekeu, J. Meunier, J. \& Rolley, E. Wetting and spreading. Rev. Mod. Phys. 81, 739-805 (2009).

4. Russell, J. T., Wang, B. Y. \& Král, P. Nanodroplet transport on vibrated nanotubes. J. Phys. Chem. Lett. 3, 353-357 (2012)

5. Duprat, C., Protière, S., Beebe, A. Y. \& Stone, H. A. Wetting of flexible fibre arrays Nature 482, 510-513 (2012). 
6. Wang, C. L. et al. Critical dipole length for the wetting transition due to collective water-dipoles interactions. Sci. Rep. 2, 358 (2012).

7. Pollack, M. G., Fair, R. B. \& Shenderov, A. D. Electrowetting-based actuation of liquid droplets for microfluidic applications. Appl. Phys. Lett. 77, 1725-1726 (2000).

8. Hayes, R. A. \& Feenstra, B. J. Video-speed electronic paper based on electrowetting. Nature 425, 383-385 (2003).

9. Srinivasan, V., Pamula, V. K. \& Fair, R. B. An integrated digital microfluidic labon-a-chip for clinical diagnostics on human physiological fluids. Lab Chip, 4, 310-315 (2004).

10. Bico, J., Roman, B., Moulin, L. \& Boudaoud, A. Adhesion: elastocapillary coalescence in wet hair. Nature 432, 690-690 (2004).

11. Zhao, Y. P., Wang, L. S. \& Yu, T. X. Mechanics of adhesion in MEMS - a review. J. Adhes. Sci. Technol. 17, 519-546 (2003).

12. Armstrong, J. E. Fringe science: Are the corollas of Nymphoides (Menyanthaceae) flowers adapted for surface tension interactions? Am. J. Bot. 89, 362-365 (2002).

13. Py, C. et al. Capillary origami: spontaneous wrapping of a droplet with an elastic sheet. Phys. Rev. Lett. 98, 156103 (2007).

14. Patra, N., Wang, B. Y. \& Král, P. Nanodroplet activated and guided folding of graphene nanostructures. Nano Lett. 9, 3766-3771 (2009).

15. Yuan, Q. Z. \& Zhao, Y. P. Precursor film in dynamic wetting, electrowetting, and electro-elasto-capillarity. Phys. Rev. Lett. 104, 246101 (2010).

16. Wang, Z. Q., Wang, F. C. \& Zhao, Y. P. Tap dance of a water droplet. Proc. R. Soc. A-Math. Phys. Eng. Sci. 468, 2485-2495 (2012)

17. Roman, B. \& Bico, J. Elasto-capillarity: deforming an elastic structure with a liquid droplet. J. Phys.: Condens. Matter 22, 493101 (2010).

18. Piñeirua, M., Bico, J. \& Roman, B. Capillary origami controlled by an electric field. Soft Matter 6, 4491-4496 (2010).

19. Guo, X. Y. et al. Two- and three-dimensional folding of thin film single-crystalline silicon for photovoltaic power applications. Proc. Natl. Acad. Sci. USA 106 20149-20154 (2009).

20. Hardy, W. B. The spreading of fluids on glass. Philos. Mag. 38, 49-55 (1919).

21. Ausserré, D., Picard, A. M. \& Léger, L. Existence and role of the precursor film in the spreading of polymer liquids. Phys. Rev. Lett. 57, 2671-2674 (1986).

22. Léger, L., Erman, M., Guinet-Picard, A. M. Ausserré, D. \& Strazielle, C., Precursor film profiles of spreading liquid drops. Phys. Rev. Lett. 60, 2390-2393 (1988).

23. Heslot, F., Fraysse, N. \& Cazabat, A. M. Molecular layering in the spreading of wetting liquid drops. Nature 338, 640-642 (1989).

24. Bortchagovsky, E. G. \& Tarakhan, L. N. Precursor film of a spreading drop of liquid crystal. Phys. Rev. B 47, 2431-2434 (1993).

25. Kavehpour, H. P., Ovryn, B. \& McKinley, G. H. Microscopic and macroscopic structure of the precursor layer in spreading viscous drops. Phys. Rev. Lett. 91 , 196104 (2003).

26. Xu, H. et al. Molecular motion in a spreading precursor film. Phys. Rev. Lett. 93 206103 (2004)

27. Webb, E. B., Grest, G. S. \& Heine, D. R. Precursor film controlled wetting of $\mathrm{Pb}$ on Cu. Phys. Rev. Lett. 91, 236102 (2003).

28. De Gennes, P. G. Wetting: statics and dynamics. Rev. Mod. Phys. 57, 827-863 (1985).

29. Neto, A. H. C., Guinea, F., Peres, N. M. R., Novoselov, K. S. \& Geim, A. K. The electronic properties of graphene. Rev. Mod. Phys. 81, 109-162 (2009).

30. Balandin, A. A. Thermal properties of graphene and nanostructured carbon materials. Nat. Mater. 10, 569-581 (2011).

31. Grantab, R., Shenoy, V. B. \& Ruoff, R. S. Anomalous strength characteristics of tilt grain boundaries in graphene. Science 330, 946-948 (2010).

32. Lee, C., Wei, X. D., Kysar, J. W. \& Hone, J. Measurement of the elastic properties and intrinsic strength of monolayer graphene. Science 321, 385-388 (2008).
33. Land, T. A., Michely, T., Behm, R. J., Hemminger, J. C. \& Comsa, G. STM investigation of single layer graphite structures produced on $\mathrm{Pt}(111)$ by hydrocarbon decomposition. Surf. Sci. 264, 261-270 (1992).

34. Li, X. S. et al. Large-area synthesis of high-quality and uniform graphene films on copper foils. Science 324, 1312-1314 (2009)

35. Koenig, S. P., Boddeti, N. G., Dunn, M. L. \& Bunch, J. S. Ultrastrong adhesion of graphene membranes. Nat. Nanotechnol. 6, 543-546 (2011).

36. Kim, K. S. et al. Large-scale pattern growth of graphene films for stretchable transparent electrodes. Nature 457, 706-710 (2009).

37. Gladstone, S., Laidler, K. \& Eyring, H. The Theory of Rate Processes (McGraw-Hill Book Company, Princeton University, 1941).

38. Derjagui, B. V. \& Churaev, N. V. Structural component of disjoining pressure. J. Colloid Interface Sci. 49, 249-255 (1974).

39. Derjaguin, B. V., Churaev, N. V. \& Muller, V. M. Surface Forces (Springer, New York, 1987).

40. Blake, T. D. \& De Coninck, J. The influence of solid-liquid interactions on dynamic wetting. Adv. Colloid Interface Sci. 96, 21-36 (2002).

41. Henle, M. L. \& Levine, A. J. Capillary wave dynamics on supported viscoelastic films: single and double layers. Phys. Rev. E 75, 021604 (2007).

42. Landau, L. D. \& Lifshitz, E. M. Fluid Mechanics (Pergamon Press, Oxford, 1987)

43. Aarts, D. G. A. L., Schmidt, M. \& Lekkerkerker, H. N. W. Direct visual observation of thermal capillary waves. Science 304, 847-850 (2004).

44. Plimpton, S. Fast parallel algorithms for short-range molecular dynamics. J. Comput. Phys. 117, 1-19 (1995)

45. Dauber-Osguthorpe, P. et al. Structure and energetics of ligand binding to proteins: escherichia coli dihydrofolate reductase-trimethoprim, a drug-receptor system. 4, 31-47 (1988).

46. Berendsen, H. J. C., Grigera, J. R. \& Straatsma, T. P. The missing term in effective pair potentials. J. Phys. Chem. 91, 6269-6271 (1987)

\section{Acknowledgments}

This work was jointly supported by the National Natural Science Foundation of China (NSFC, Grant Nos. 11072244 and 11021262), the Instrument Developing Project of the Chinese Academy of Sciences (Grant No. Y2010031) and the Key Research Program of the Chinese Academy of Sciences (Grant No. KJZD-EW-M01).

\section{Author contributions}

X.Y.Z. performed most of the numerical simulations. Y.P.Z. and X.Y.Z. carried out most of the theoretical analysis. Q.Z.Y. carried out some numerical simulations and theoretical analysis. Y.P.Z., X.Y.Z. and Q.Z.Y. jointly contributed the ideas and wrote the paper. All authors discussed the results and commented on the manuscript.

\section{Additional information}

Supplementary information accompanies this paper at http://www.nature.com/ scientificreports

Competing financial interests The authors declare no competing financial interests. License: This work is licensed under a Creative Commons Attribution-NonCommercial-NoDerivs 3.0 Unported License. To view a copy of this license, visit http://creativecommons.org/licenses/by-nc-nd/3.0/

How to cite this article: Zhu, X., Yuan, Q. \& Zhao, Y. Capillary wave propagation during the delamination of graphene by the precursor films in electro-elasto-capillarity. Sci. Rep. 2, 927; DOI:10.1038/srep00927 (2012). 